\title{
No Right to Speak? The Relationship between Object Naming and Semantic Impairment: Neuropsychological Evidence and a Computational Model
}

\author{
M. A. Lambon Ralph ${ }^{1}$, J. L. McClelland ${ }^{2}$, K. Patterson ${ }^{3}$, C. J. Galton ${ }^{4}$, \\ and J. R. Hodges ${ }^{3,4}$
}

\begin{abstract}
The processes required for object naming were addressed in a study of patients with semantic dementia (a selective decline of semantic memory resulting from progressive temporal lobe atrophy) and in a computational model of single-word production. Although all patients with semantic dementia are impaired in both single-word production and comprehension, previous reports had indicated two different patterns: (a) a parallel decline in accuracy of naming and comprehension, with frequent semantic naming errors, suggesting a purely semantic basis for the anomia and (b) a dramatic progressive anomia without commensurate decline in comprehension, which might suggest a mainly postsemantic source of the anomia. Longitudinal data for 16 patients with semantic dementia reflected these two profiles, but with the following additional important specifications: (1) despite a few relatively extreme versions of one or other profile, the full set of cases formed a continuum in the extent of anomia for a
\end{abstract}

\section{INTRODUCTION}

Speech production is typically initiated by the meaning of the intended utterance or word. This is true not only for spontaneous speech, but also for single-word production as in naming a seen object, where visual input first activates the corresponding conceptual knowledge that then initiates spoken output (Morton, 1985). All theories of word production assume that disruption to conceptual knowledge will yield anomia, an assumption that is supported by neuropsychological investigations of semantically impaired patients. In this paper, by the combined use of results from neuropsychology, neuroanatomy, and a computational model, we consider the proposal that semantic abnormality might be a sufficient explanation of anomia even in many cases that have previously been thought to require a different account.

\footnotetext{
${ }^{1}$ University of Bristol, Bristol, UK, ${ }^{2}$ Center for the Neural Basis of Cognition, Pittsburgh, ${ }^{3}$ MRC Cognition and Brain Sciences Unit, Cambridge, UK, ${ }^{4}$ Addenbrooke's Hospital, Cambridge, UK
}

given degree of degraded comprehension; (2) the degree of disparity between these two abilities was associated with relative asymmetry in laterality of atrophy: a parallel decline in the two measures characterized patients with greater rightthan left-temporal atrophy, while disproportionate anomia occurred with a predominance of atrophy in the left-temporal lobe. In an implemented computational model of naming, semantic representations were distributed across simulated left- and right-temporal regions, but the semantic units on the left were more strongly connected to left-lateralized phonological representations. Asymmetric damage to semantic units reproduced the longitudinal patient profiles of naming relative to comprehension, plus additional characteristics of the patients' naming performance. On the basis of both the neuropsychological and computational evidence, we propose that semantic impairment alone can account for the full range of word production deficits described here.

Deficits of semantic memory can result from a variety of causes that typically involve damage to one or both temporal lobes, including Alzheimer's disease (Hodges \& Patterson, 1995), cerebral vascular accident (CVA: Chertkow, Bub, Deaudon, \& Whitehead, 1997), head injury (Bub, Black, Hampson, \& Kertesz, 1988), herpes simplex virus encephalitis (HSVE: Kapur et al., 1994), and semantic dementia (Hodges, Patterson, Oxbury, \& Funnell, 1992). Semantic dementia, which represents the temporal variant of the spectrum of frontotemporal dementias (Hodges, 2000; Snowden, Neary, \& Mann, 1996), results from progressive, circumscribed atrophy of the temporal lobes. Recent techniques designed to quantify the degree of structural abnormality in various brain regions indicate that the atrophy in semantic dementia is most pronounced in the anterior, inferior, and lateral aspects of the temporal lobes (Galton et al., submitted; Mummery, Patterson, Wise, Price, \& Hodges, 1999; Mummery, et al., 2000). With the exception of a few patients investigated at the very earliest stages of the disease, the atrophy nearly always involves both temporal lobes, although its distribution is often 
strongly asymmetric, with the majority of reported cases characterized by more extensive atrophy on the left.

Relative to other etiologies, semantic dementia has two clear advantages for the study of degraded conceptual knowledge and its impact on speech production: selective and progressive impairment of semantic memory. Warrington's (1975) seminal paper was the first to highlight the selective nature of semantic impairment in these cases; and some years later, this observation was encapsulated by Snowden, Goulding, and Neary (1989) in the now commonly accepted name for the disorder-semantic dementia (Saffran \& Schwartz, 1994; Hodges et al., 1992). Until very late stages in the disease, the patients have impaired comprehension and pronounced anomia without any of the significant deficits that often accompany semantic impairment in other aetiologies; these preserved cognitive domains include day-to-day memory, orientation, nonverbal problem solving, perceptual and spatial skills, and, within the language domain, syntactic and phonological processing. The language pattern of anomia with relatively good syntax and phonology also gives this disorder yet another appropriate, if less encompassing, label: progressive fluent aphasia. With regard to the other main advantage for the study of impaired conceptual knowledge, the progressive nature of the disorder enables comparison of performance not only cross-sectionally across a group of patients but also longitudinally within individuals. Such analyses of the progressive degradation of conceptual knowledge are yielding some important insights into the organization of semantic memory and its relationship to other aspects of memory and language (Patterson \& Hodges, 2000).

Research on semantic dementia has revealed highly specific and theoretically informative deficits in tasks such as reading aloud (Graham, Hodges, \& Patterson, 1994; Chiacchio, Grossi, Stanzione, \& Trojano, 1993), auditory short-term memory (Knott, Patterson, \& Hodges, 1997), transforming the present tense of an English verb to its past tense (Patterson, McClelland, Lambon Ralph, \& Hodges, in press) and recognition memory (Graham, Simons, Pratt, Patterson, \& Hodges, 2000). Although this research suggests a clear role for conceptual knowledge in these domains, in each, the influence is limited; this is because nonsemantic processes are sufficient to achieve a considerable degree of success in these tasks. For example, the principal processes in reading aloud involve translation from orthographic to phonological representations, without reliance on word meaning (Plaut, McClelland, Seidenberg, \& Patterson, 1996). In speech production and comprehension, by contrast, conceptual knowledge takes center stage. Given the greater sensitivity to damage of expressive over receptive tasks (Gainotti, Silveri, Daniele, \& Giustolisi, 1995), it is unsurprising to find that word-finding difficulties tend to be more prominent than the accompanying comprehension deficit. The typical presenting feature for patients with semantic dementia is, therefore, anomia, although impaired comprehension is usually uncovered by careful testing, even at an early phase and-as will be demonstrated below - always at more advanced stages.

The direct relationship between semantic impairment and anomia was highlighted in a longitudinal study of the naming performance of $\mathrm{JL}$, a patient with semantic dementia (Hodges, Graham, \& Patterson, 1995). JL was asked to name a corpus of 260 line drawings of familiar objects (the pictures from Snodgrass \& Vanderwart, 1980) on four occasions over an 18-month period. In this period, his naming accuracy on this set fell from 36\% to just under $1 \%$, a precipitous decline that was coupled to a similar drop in comprehension. Progressive semantic deterioration was emphasized as the locus of JL's anomia by the pattern of his naming errors. Initially, these were dominated by coordinate semantic errors (e.g., ostrich $\rightarrow$ "swan", peacock $\rightarrow$ "duck"), then by superordinate responses (e.g., ostrich $\rightarrow$ "bird," peacock $\rightarrow$ "bird"), and ultimately by the name of the appropriate broadest domain (e.g., ostrich $\rightarrow$ "animal") or of high-frequency, prototypical exemplars from this domain (e.g., peacock $\rightarrow$ "cat"). From both this pattern of error type and the coupled decline in accuracy of naming and comprehension, JL's longitudinal performance suggested that speech production-as measured in this case by picture naming-can directly reflect the status of conceptual knowledge.

By contrast, a different longitudinal profile was reported for case FM. FM was initially described as a case of semantic dementia (Hodges et al., 1992) on the basis of severe anomia and mild semantic impairment with a highly characteristic picture of inferolateral left-temporal atrophy; but investigations over the next few years revealed a pattern described as "progressive pure anomia" (Graham, Patterson, \& Hodges, 1995): whereas FM's severe anomia became even more pronounced, her performance on various comprehension tests remained fairly constant, with scores only minimally outside the normal range. Like other patients with semantic dementia, FM had excellent single-word phonology-i.e., normal single-word repetition, good nonword reading and an absence of phonological errors in speech. Graham et al., therefore, concluded that FM's profound and worsening anomia could not be explained by deficits in either the phonological or the semantic domain, thus required an interpretation of reduced transmission of activation between semantic and phonological representations. Subsequently, FM's comprehension also deteriorated significantly (see Graham, Patterson, \& Hodges, in press; Tyler, Moss, Patterson, \& Hodges, 1997), reestablishing the pattern expected in semantic dementia: both severe anomia and poor comprehension without global cognitive decline. 
The two longitudinal profiles shown by JL and FM give rise to a conundrum that mirrors the central issue of this study. One interpretation would be that the temporal variant of frontotemporal dementia should be split into two categories: progressive anomia and semantic dementia (Graham, Patterson, \& Hodges, 1998). In the former, the initial anomia of patients like FM, which apparently could be ascribed to neither a semantic nor a phonological impairment, would require an impaired locus between these two domains; in many models of speech production (e.g., Dell \& O'Seaghdha, 1992; Levelt, 1992), this level would correspond to lemmas or abstract, word-specific representations. An alternative interpretation would emphasize that the difference between FM and JL was more one of degree than of type. Both had impaired comprehension but more pronounced word-finding difficulties; it is only the degree of the disparity between these two deficits that differentiates the two patients. Even then, since FM's comprehension subsequently declined to a level commensurate with her profound anomia, the two patterns are differentiated only at the early and middle stages of progression.

A factor that might play a key role in an explanation for the two longitudinal profiles is the nature of the connectivity between the semantic system and the processes required for speech production. Suppose that conceptual knowledge is supported by a large, distributed neural system, but that the phonological representations for speech production are (i) more tightly localized and (ii) activated primarily by connections from neuroanatomically closer sections of the full semantic network. In this case, the degree of anomia resulting from a purely semantic deficit would vary with the extent and location of the damage within the semantic neural system. We will show in this article that if these assumptions are embodied within a computational model, then the key aspects of the patients' data can be reproduced by varying the degree and pattern of damage to the units that support the conceptual representations, without any additional locus of impairment. Results from the simulations are preceded by longitudinal data on tests of naming and comprehension for 16 individuals with semantic dementia. These neuropsychological data are used to address two major questions: (a) Are the distinctive longitudinal profiles of JL and FM reproduced by other individual cases? (b) Is there an independent measure, which predicts the two profiles and thereby helps to illuminate the basis for the difference?

\section{BEHAVIORAL DATA}

Four example, longitudinal profiles-those for FM and $\mathrm{JL}$ as described, plus two additional cases, SC and $\mathrm{VH}-$ are shown in Figure 1. Each point represents one patient's scores at a specific time period on the tests of picture naming and word-picture matching, the latter serving as a measure of comprehension. A number of observations are worthy of note. First, the degree of anomia was always greater than the corresponding comprehension impairment in all cases, i.e., each individual profile falls below the diagonal dotted line denoting equivalent performance, and typically by a greater amount than would be expected solely on the basis that chance responding can yield a small number of correct responses for word-picture matching but none for naming. Secondly, the profiles for cases SC and $\mathrm{VH}$ largely reproduce the longitudinal patterns noted above for patients FM and JL, respectively. FM's anomia is strikingly demonstrated in Figure 1a. Even at the initial presentation, FM's mild comprehension impairment (44/48) was accompanied by a severe anomia (7/48). Over subsequent testing sessions, her ability to name these 48 pictures first dropped to zero, followed some time later by a relentless decline in
Figure 1. Four patients' longitudinal profiles of picture naming versus word-picture matching.

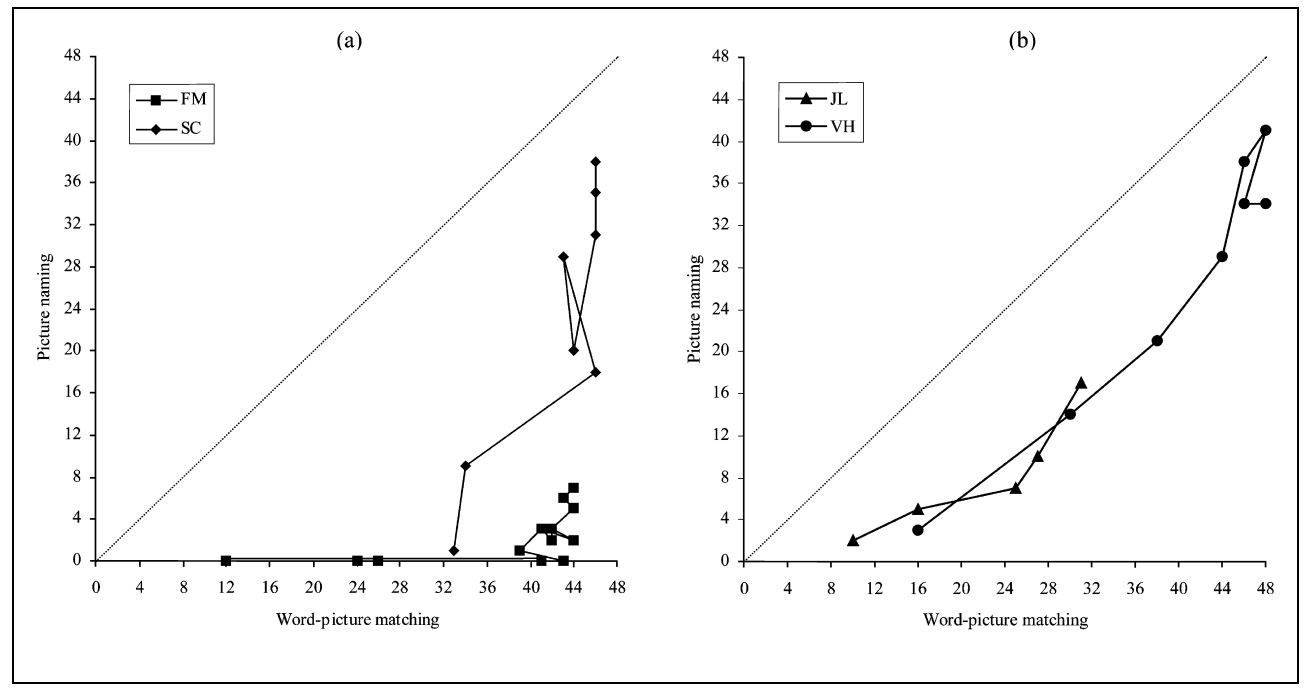




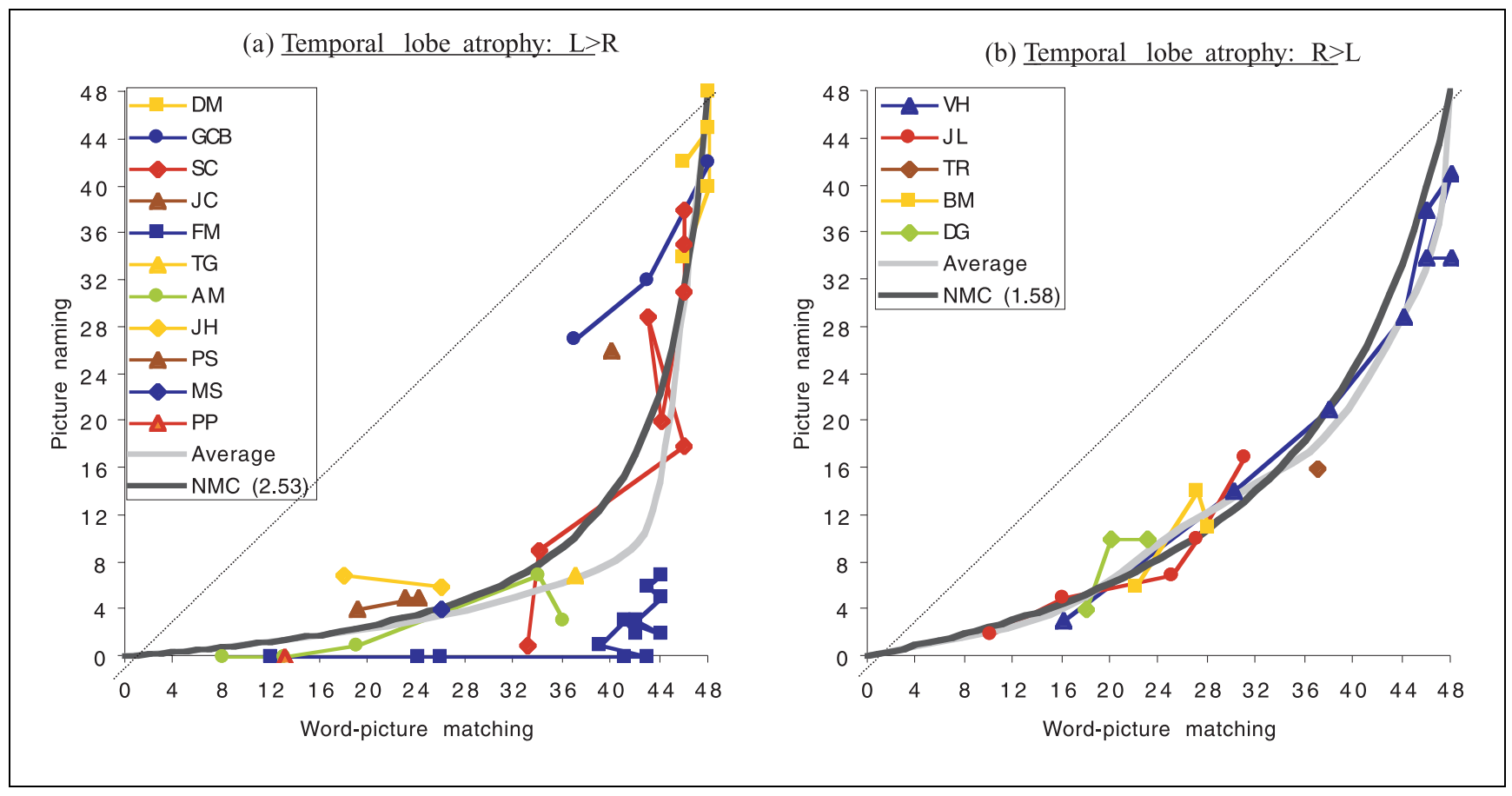

Figure 2. Individual and grouped naming and word-picture matching data split by the patients' relative asymmetry of temporal lobe atrophy and corresponding mean NMC curves.

comprehension. ${ }^{1}$ Normal control subjects score at, or very close to, ceiling on both tasks, indicating that much of the decline in FM's word-finding abilities had already occurred prior to her initial assessment. This undocumented early phase of progressive anomia for FM may have resembled that demonstrated by patient SC (see Figure 1a), who presented with a very mild comprehension deficit (46/48) plus anomia (35/ 48). Over the next five testing sessions, SC's performance on the word-picture matching task remained relatively constant, but her naming dropped precipitously (to 18/48). Then, as her ability to name declined to floor level, her comprehension began to deteriorate as well.

JL's performance on this set of items is shown in Figure 1b. When first tested, he demonstrated poor naming (17/48) and impoverished comprehension (31/ 48), and his performance on these two tasks deteriorated in parallel over the next four sessions. A similar but fuller longitudinal profile was collected from patient $\mathrm{VH}$. As can be seen in Figure 1b, after an initial improvement possibly attributable to a practice effect, VH's naming and comprehension declined largely in concert.

The full set of data from 16 cases is shown in Figure 2. Here, the patients are assigned to the two sides of the figure on the basis of the relative asymmetry of the temporal lobe atrophy, because initial observations suggested that this might be a relevant factor. The progressive anomic pattern of patients FM and SC characterized those individuals with greater left- than right-temporal lobe atrophy, hereafter denoted as L > R (see Figure 2a).
By contrast, the coupled decline in naming and comprehension that described cases like JL and $\mathrm{VH}$ was associated with greater right- than left-temporal lobe atrophy, hereafter R > L (Figure 2b). The distributions of longitudinal profiles were compared for the two sets of patients using two different techniques (see Methods). Analyses of the complete set of naming data, based on a simple binary classification of the patients as having greater L $>$ R or R $>$ L atrophy, produced significant effects of both comprehension (naming scores reduced as comprehension declined, $F(4,55)=38.6, p<.001)$ and laterality (naming was worse for the $\mathrm{L}>\mathrm{R}$ patients, $\mathrm{F}(1,55)=9.54, p=.003)$. Using a measure that we have called the naming-matching correspondence (NMC) curve (see Methods), each individual profile was reduced to a single parameter $A$ that reflects the degree of disparity between naming and word-picture matching. Analysis of the fitted values of the parameter $A$ revealed that $A$ was significantly lower for those patients with more right-temporal (mean $A=1.58$ ) than left-temporal (mean $A=2.53$ ) lobe atrophy (Mann-Whitney $U$, exact $p=.05)$.

Further analyses utilized the more detailed rated volumes of temporal lobe structures calculated for 14 of the 16 patients. The degree of global temporal atrophy correlated negatively with both the patients' word-picture matching score $(r=-.62, p=.01)$ and naming accuracy $(r=-.58, p=.02)$; these behavioral measures themselves were, as expected, highly intercorrelated $(r=.85, p<.001)$. Importantly, the degree of asymmetry correlated with the parameter $A$ estab- 


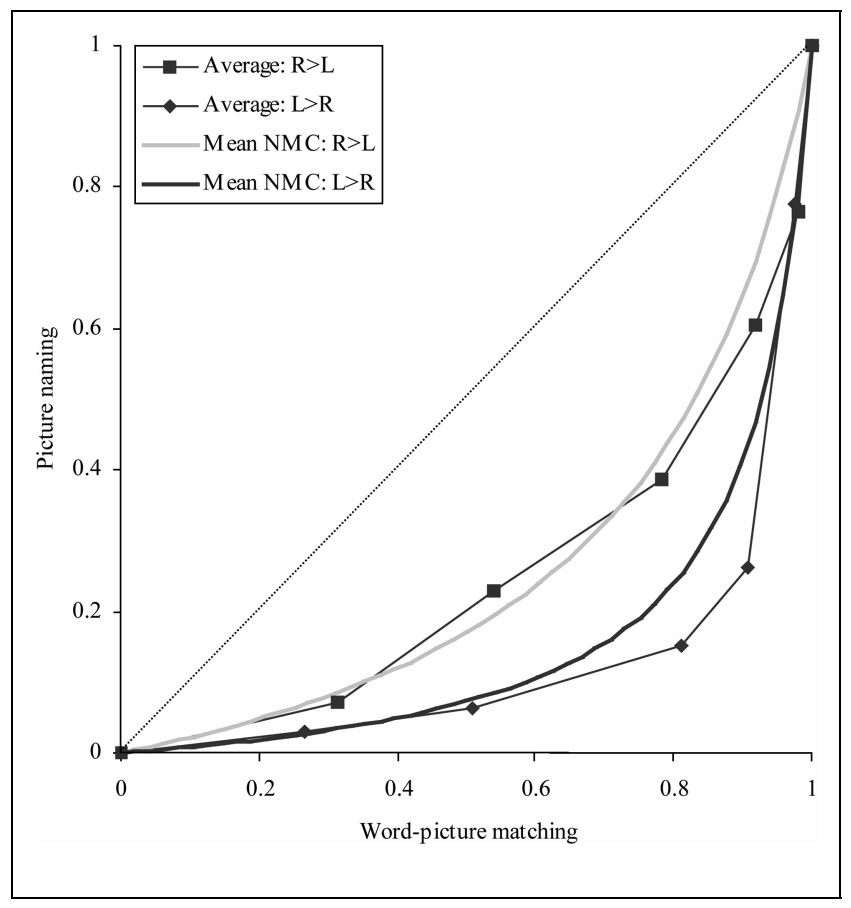

Figure 3. Grouped naming and word-picture matching data split by the patient's relative asymmetry of temporal lobe atrophy and corresponding mean NMC curves.

lished for each patient (partial correlation after controlling for the effect of global temporal lobe atrophy, $r=.64, p=.01$ ), indicating that as the asymmetry value increased (positive $=$ more left-sided atrophy, negative $=$ more right-sided atrophy), the extremity of the curvature in the NMC function also increased.
Figure 3 summarizes the longitudinal profiles for the two patient groups, in terms of both the grouped data and the mean NMC curves. The degree of anomia was always greater than the accompanying comprehension deficit in all patients, and the drop in naming performance was particularly pronounced early in the course of the disease, supporting the clinical observation that word-finding difficulties are the most striking symptom at presentation for these patients. These averaged figures merely illustrate in a more transparent fashion what was demonstrated by Figure 2 and the analyses described above, namely, that the disparity between naming and comprehension was more marked for the $\mathrm{L}>\mathrm{R}$ patients, whereas more extensive right-sided atrophy yielded a closer coupling of naming and comprehension performances over time.

The two sets of patients also differed with regard to the pattern of naming errors produced. Table 1 shows the overall accuracy for naming and word-picture matching collapsed across time for the two groups, as well as the distribution of naming error types. The differential disparity between comprehension and naming accuracy for the two groups is reflected by equivalent naming performance associated with a significantly lower score for word-picture matching for the $\mathrm{R}>\mathrm{L}$ cases. The $\mathrm{L}>\mathrm{R}$ patients produced more omission or no response errors and were more likely to provide functional descriptions of the pictured items (e.g., lobster $\rightarrow$ "an animal but you can eat it"; rocking chair $\rightarrow$ "one of those seats you sit on that goes backwards and forwards"). Visual descriptions (e.g., zebra $\rightarrow$ "animal ... with black pieces across") were rare in both groups.

Table 1. Type and Frequency of Naming Errors for the Two Patient Groups

\begin{tabular}{lccc}
\hline & \multicolumn{2}{c}{ Asymmetry of Temporal Lobe Atrophy } & Significance \\
\cline { 2 - 3 } & Left $>$ Right & Right $>$ Left & p (t value $)$ \\
\hline Number of observations & 38 & 19 & $.03(2.28)$ \\
Word-picture matching & $37.2(11.0)$ & $29.9(12.2)$ & \\
Naming & & & $n . s$. \\
Accuracy $^{\mathrm{a}}$ & $15.7(15.9)$ & $16.3(12.7)$ & \\
Error type $^{\mathrm{a}}$ & & & $.003(3.15)$ \\
$\quad$ Omission & $19.0(15.2)$ & $7.6(5.89)$ & $.001(3.48)$ \\
$\quad$ Functional description & $6.6(6.65)$ & $1.2(1.69)$ & $n . s$. \\
$\quad$ Visual description & $.29(.65)$ & $.53(.96)$ & $n . s$. \\
$\quad$ Superordinate & $3.1(5.53)$ & $3.6(3.34)$ & $<.001(-8.90)$ \\
Coordinate & $2.9(3.27)$ & $14.6(6.71)$ & $<.001(-5.28)$ \\
$\quad$ Visual errors & $.11(.39)$ & $2.2(2.42)$ & \\
\hline
\end{tabular}

n.s. $=$ not significant.

${ }^{\mathrm{a}}$ Mean (standard deviation): maximum score $=48$. 
Slightly higher but equivalent numbers of superordinate errors (e.g., hen $\rightarrow$ "bird"; violin $\rightarrow$ "musical instrument") were produced by both patient groups. There was a significant difference, however, in the rate of semantic coordinate errors (e.g., ostrich $\rightarrow$ "swan"; helicopter $\rightarrow$ "airplane"), which were produced more often by the $\mathrm{R}>\mathrm{L}$ patients. There was also a small but reliable difference in the same direction for the rate of purely visually related errors (e.g., harp $\rightarrow$ "fence").

As noted above for the relationship between comprehension and naming accuracy, the difference between the two groups in terms of the types of naming error produced is graded rather than categorical. All patients produced a mixture of omissions, functional descriptions, and coordinate and superordinate semantic errors, but the exact proportions varied systematically in line with the relative asymmetry in temporal lobe atrophy. This observation was corroborated by further analyses using the detailed rated volumes of temporal lobe structures. Production of coordinate semantic errors correlated negatively with the measure of asymmetry (after controlling for the effect of global temporal lobe atrophy) indicating that semantic errors increased as the balance of atrophy became more pronounced in the right-temporal lobe. This was true whether the measure of semantic errors adopted was the raw number $(-.93$, $p<.001)$ or the number of semantic errors as a proportion of total errors $(-.79, p<.001)$.

\section{Summary}

The longitudinal analysis of a cohort of patients with semantic dementia revealed two broad patterns of progressive decline in the measures of interest here. In one set of individuals, the decline in object-naming accuracy was closely linked to a deteriorating level of comprehension (as measured by word-picture matching), and the most common type of naming error was to provide the label appropriate to another exemplar from the same semantic category. In the other set of patients, the initial part of the longitudinal profile was dominated by a swiftly progressive anomia with only a small accompanying decline in comprehension. The latter part of their decline revealed substantial deterioration in comprehension, too, though by this time picture-naming performance had reached floor level. Naming errors in this second group were most likely to be omissions.

One explanation for the two longitudinal profiles would propose that these patterns reflect two different functional deficits. By this account, the first set of patients would have the expected pattern for semantic dementia-dissolution of conceptual knowledge leading to poor comprehension, word-finding difficulties and the production of semantically related errors. The second group of patients would have a postsemantic deficit leading to their progressive anomic profile (Papagno \&
Capitani, submitted; Graham et al., 1995, 1998). Such an impairment might also explain why the most common naming error in this group was a failure to respond: One could hypothesize that although pictures were able to access much of the corresponding conceptual knowledge, a major deficit at a stage subsequent to semantic representations would prevent sufficient activation from arriving at the level phonological representations. This "two-impairment" hypothesis, however, has several limitations. All differences between the patient groups were ones of degree rather than type. Longitudinal profiles all began and especially ended in very similar ways; and rather than the patterns falling into two neat, nonoverlapping groups, there was a continuum of profiles from the apparently semantically driven anomia of patients like JL and $\mathrm{VH}$ to the disproportionate progressive anomia of FM and SC. Another unsatisfactory part of the two-impairment hypothesis is that it offers no basis for predicting which patients should fall into which group: Given a single data point near the upper right corner of Figure 3, there is no independent factorpsychological, neuroanatomical, or otherwise-that would enable one to predict that its subsequent longitudinal trajectory should fit one or other of the two patterns.

With a series of computational simulations, we shall outline an alternative proposal that provides a better solution to the puzzle posed by these data. This hypothesis rests upon four central assumptions: (1) Progressive semantic impairment is the one and only key deficit in all these cases. (2) Conceptual representations are supported by bilateral temporal lobe structures, but phonology is strongly left lateralized. (3) Two

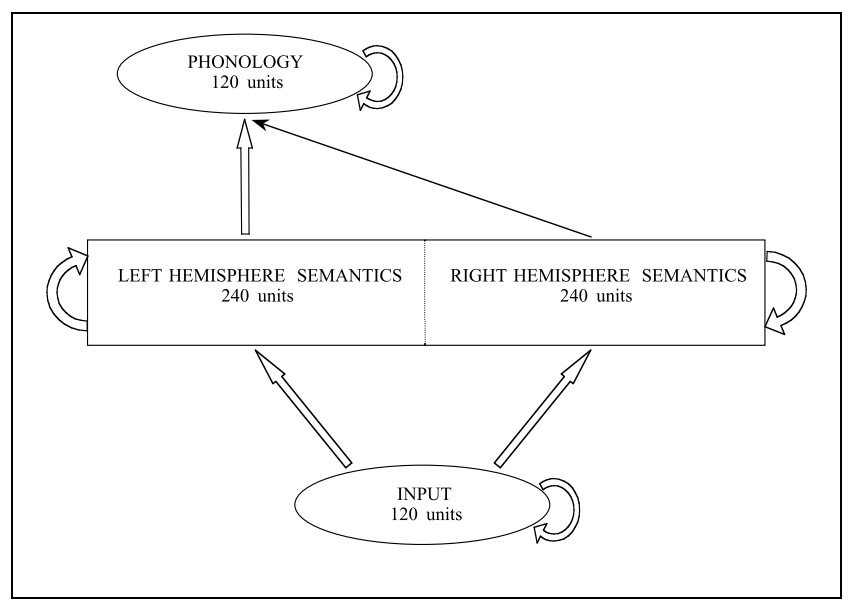

Figure 4. Architecture used for simulating the comprehension and naming performance of patients with semantic dementia. The direction of the arows denotes the type of connectivity within and betweenlayers. The thickness of the arrows represents the relative learning rate applied to each set of connections (i.e., equivalent for all connections within the network except for those between the right hemisphere semantics and phonology. 
communicating functional systems supported by regions on the same side of the brain will be more tightly coupled than if the supporting neural substrates are in different hemispheres (Plaut, 1999). (4) The degree of asymmetry in an individual patient's bilateral temporal lobe atrophy determines the shape of the longitudinal curve relating the degree of anomia to the level of comprehension deficit.

\section{SIMULATIONS}

\section{Part 1: The Effect of Left-lateralized Phonological Representations}

The architecture of the simulations reported here is shown in Figure 4. A number of key assumptions were embedded within this network. As noted in the Introduction, most theories of word production assume that picture input cannot directly activate phonological forms. Rather the picture accesses its corresponding conceptual knowledge, which is used in turn to drive speech production. Conceptual representations were encoded within a single distributed "semantic" system that was assumed to be supported by bilateral temporal lobe structures in the brain. For purposes of simplification, there was no differentiation in the type of semantic information encoded on the "left" or "right" hemisphere semantic units.

Once the network had been trained to produce the correct "semantic" and "phonological" representations from a given input pattern (see Methods for details of training procedure), it was subjected to simulated semantic damage in the form of bilateral but asymmetric "lesions." The simulated damage was concentrated primarily either on the left or right semantic units for all degrees of impairment. This resulted in two projected profiles for relatively extreme left- or right-sided damage. These can be thought of as establishing the boundaries of an envelope of possible longitudinal profiles of naming versus comprehension in patients with semantic dementia who vary in the exact proportion of left- and right-temporal lobe atrophy.

The effect of strongly left-lateralized phonological representations was the subject of the first set of simulations. Effective laterality was introduced into the model by connecting the phonological units more strongly to the left than the right semantic units. This was achieved by reducing the learning rate on the connections between the right semantic and phonological units. The effect of manipulating the learning rate on these connections is shown in Figure $5 \mathrm{~A}$ and $\mathrm{B}$, for
Figure 5. (A) Manipulating the learning rate on the connections between the right semantic and phonological units: effect on naming. (B) Manipulating the learning rate on the connections between the right semantic and phonological units: effect on comprehension. Right $>$ Left-greater damage to right than left semantic units. Left > Right - greater damage to left than right semantic units. * Scaling factor - relative strength of the learning rate parameter for the connections between the right hemisphere semantic and phonological units.

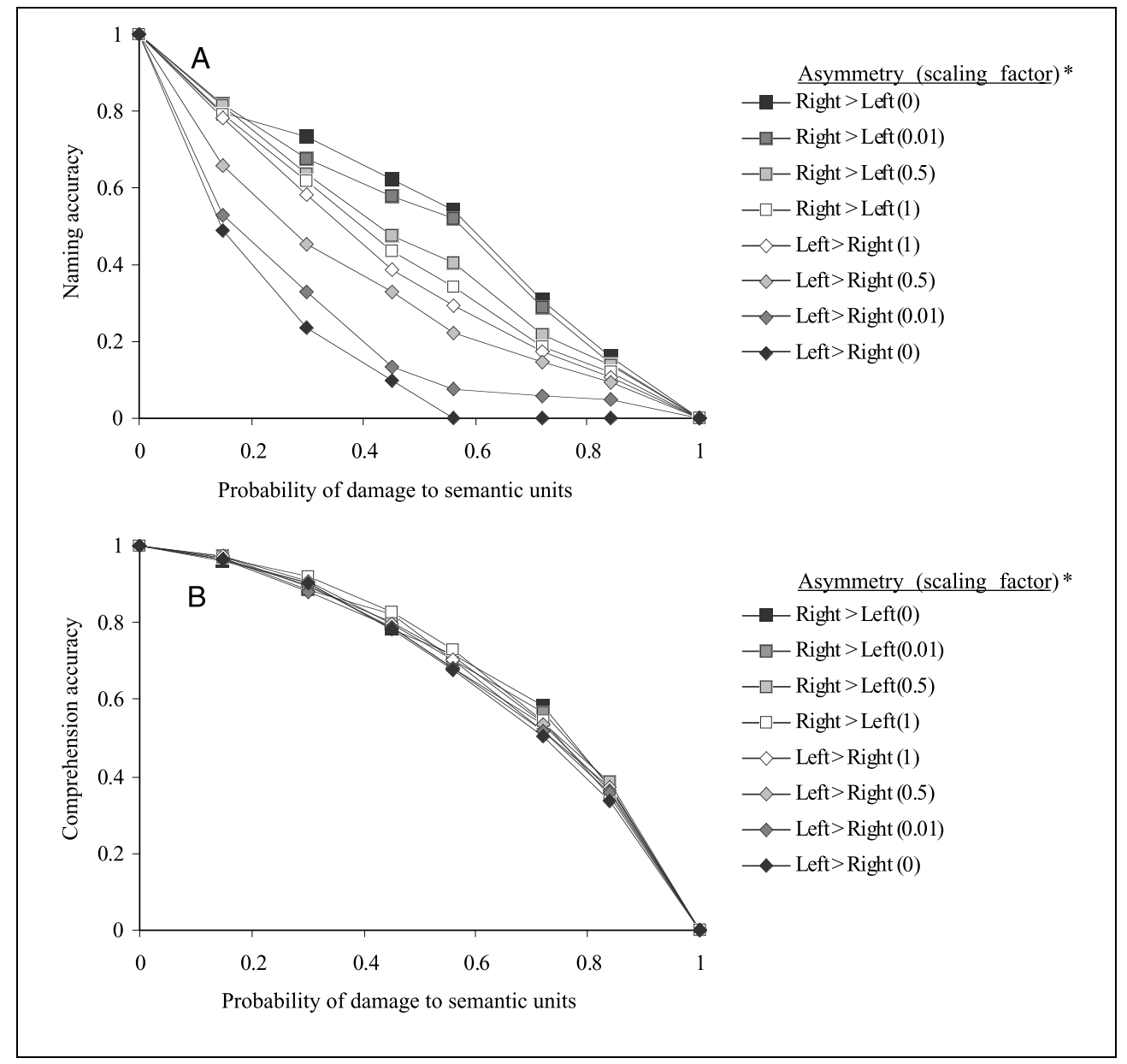




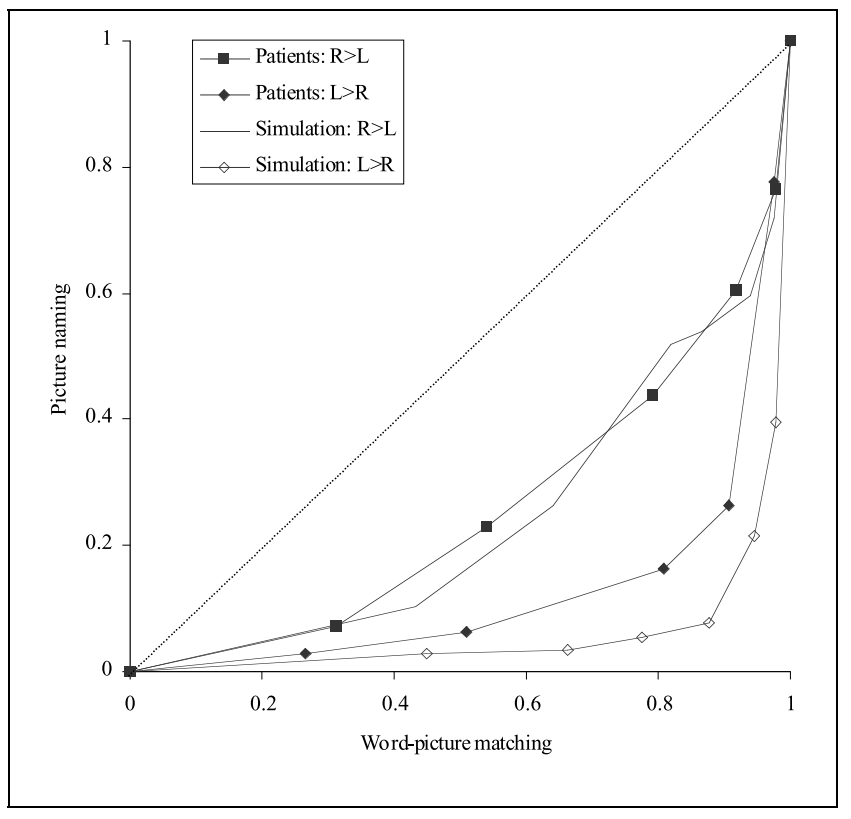

Figure 6. Comprehension versus naming: direct comparison between simulation and grouped patient data. Patients: R $>$ L-Patients with asymmetric right temporal lobe atrophy. Patients: $\mathrm{L}>\mathrm{R}$-Patients with asymmetric left temporal lobe atrophy. Simulation: $\mathrm{R}>\mathrm{L}-$ Simulation with more right than left semantic unit damage. Simulation: $\mathrm{L}>\mathrm{R}-$ Simulation with more left than right semantic unit damage.

naming and comprehension performance, respectively. The fact that this manipulation had no effect on comprehension (Figure 5B) is unsurprising given that the semantic representations are unbiased and trained in an equivalent manner for both sides of the network. Naming performance, by contrast, was clearly affected by variations in the learning rate on connections from right semantic elements to phonology. As this rate was reduced (see Figure $5 \mathrm{~A}$ ), accurate naming performance became both more reliant on the integrity of the left semantic units and less dependent upon the right semantic units. When damage was concentrated more on the right than left semantic units $(\mathrm{R}>\mathrm{L})$, a lower learning rate led to relatively better naming performance at each level of damage (the curve moves upwards). The converse was true when damage was focused more upon the left than right semantic units $(\mathrm{L}>\mathrm{R})$. This first set of simulations, utilizing bilateral semantic but left-lateralized phonological representations, begins to provide a match to the performance seen in semantic dementia. Irrespective of the level of overall damage, a lesion to the semantic units resulted in greater anomia than comprehension impairment. The rate of decline in naming performance was most pronounced with small amounts of semantic unit damage. The degree of disparity between comprehension and naming performance was governed by the relative asymmetry of the damage to the semantic units. When damage was concentrated primarily upon the left side, naming accuracy was much more sensitive to small amounts of damage. Ultimately, both distributions of damage ended with extremely poor comprehension and naming performance.

\section{Part 2: A Direct Comparison of Patients and Simulation}

One of the previous simulations served as the basis for a direct comparison between the behavioral data of the patients and the output of the network. The model used a 0.01 scaling factor for the learning rate on the connections from right semantic to phonological units. The profile for this simulation with $\mathrm{R}>\mathrm{L}$ damage provided an extremely close match to the patients with $\mathrm{R}>\mathrm{L}$ temporal lobe atrophy, for the group as a whole (Figure 6), as well as for the individual cases JL and VH (Figure 7b). ${ }^{2}$ The trajectory for the simulation with $\mathrm{L}>\mathrm{R}$ semantic unit damage was also very similar to, albeit slightly more
Figure 7. Comprehension versus naming: direct comparison between simulation and four individual patients. a) Simulation: $\mathrm{L}>\mathrm{R}$-Simulation with more left than right semantic unit damage. b) Simulation: $\mathrm{R}>\mathrm{L}$-Simulation with more right than left semantic unit damage.

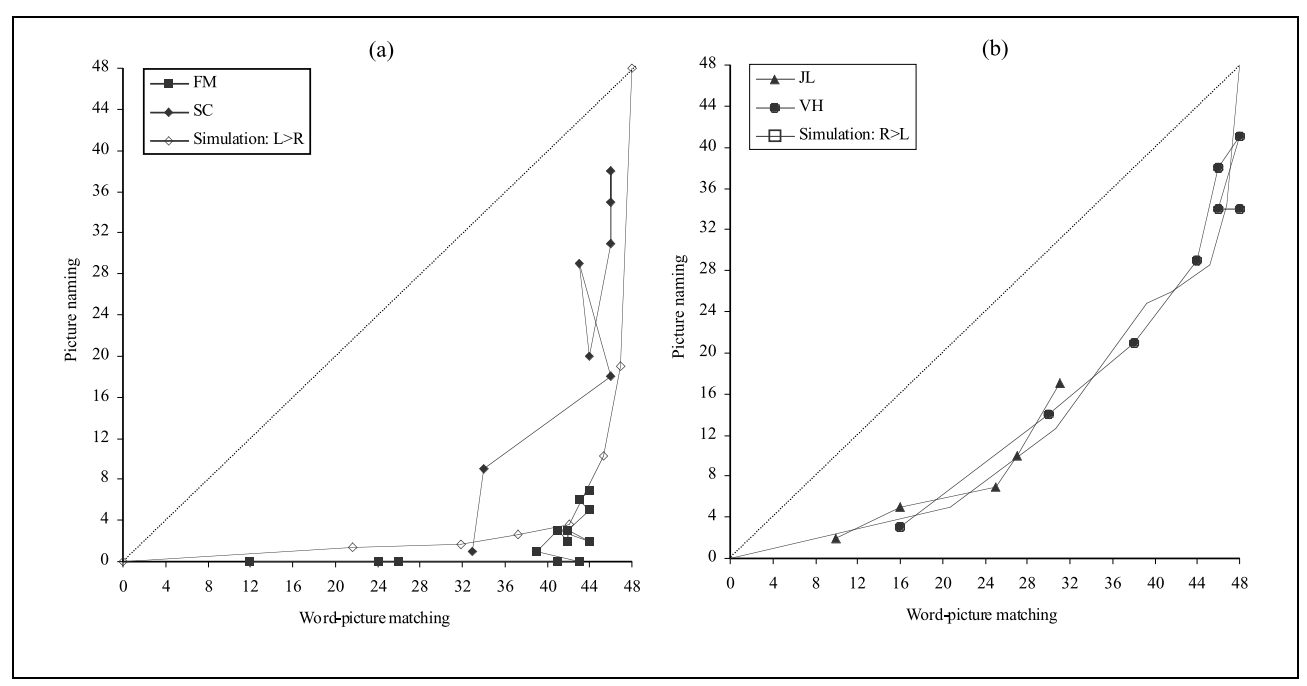


extreme than, the corresponding patient data for the entire $L>R$ group. This extreme profile falls close to the data for the two most extreme patients, FM and SC (Figure 7a).

Considering next the semantic category coordinate errors, we see in both the simulations (Figure 8A) and the patients (Figure $8 \mathrm{~B}$ ) that the proportion of such errors declines as the overall comprehension impair-

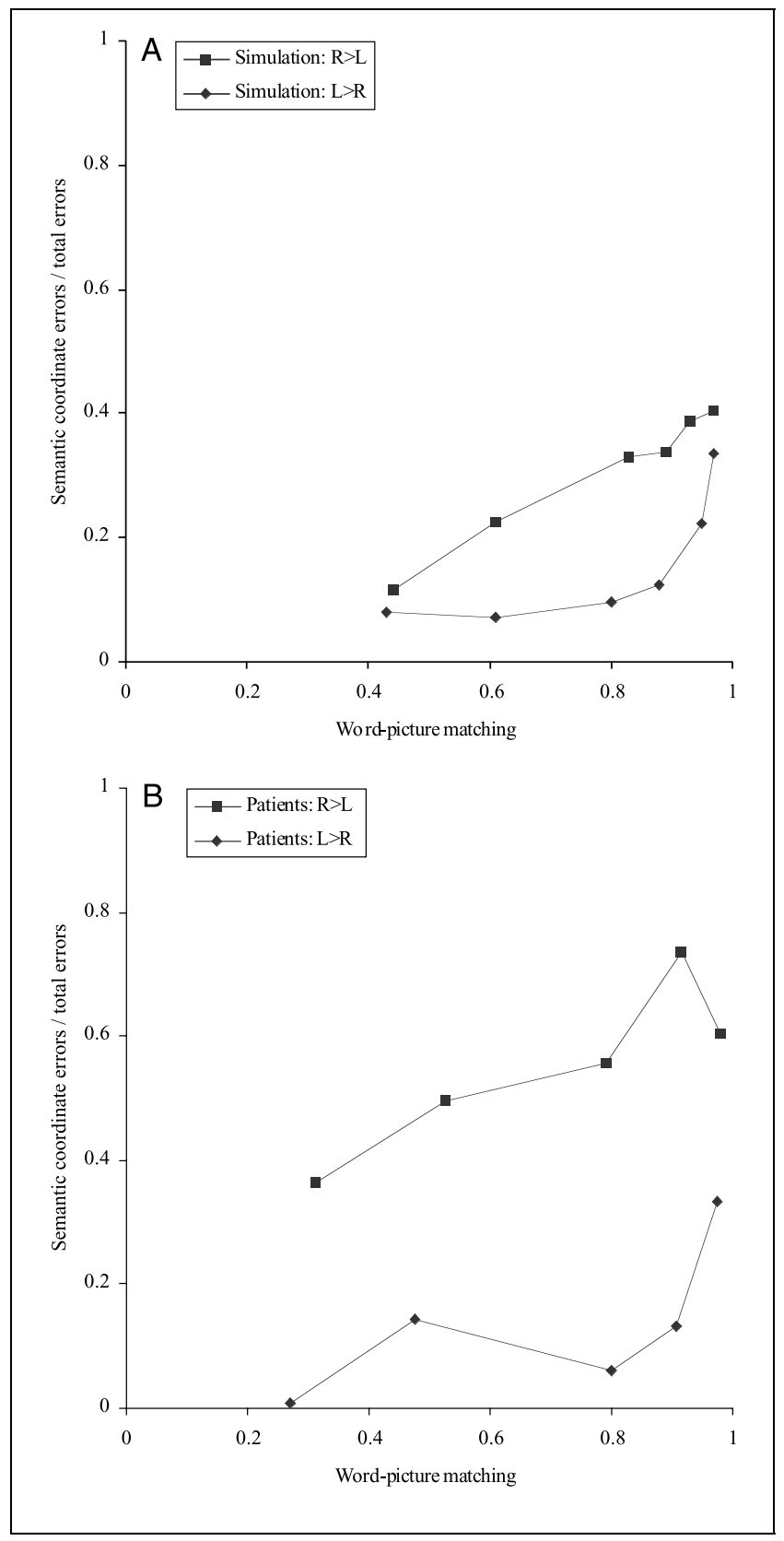

Figure 8. (A) Rate of semantic errors observed in the simulation. Simulation: $\mathrm{R}>\mathrm{L}-$ Simulation with more right than left semantic unit damage. Simulation: $\mathrm{L}>\mathrm{R}$-Simulation with more left than right semantic unit damage. (B) Rate of (coordinate) errors in the grouped patient data. Patients: $\mathrm{R}>\mathrm{L}$-Patients with asymmetric right temporal lobe atrophy. Patients: $\mathrm{L}>\mathrm{R}$-Patients with asymmetric left temporal lobe atrophy.

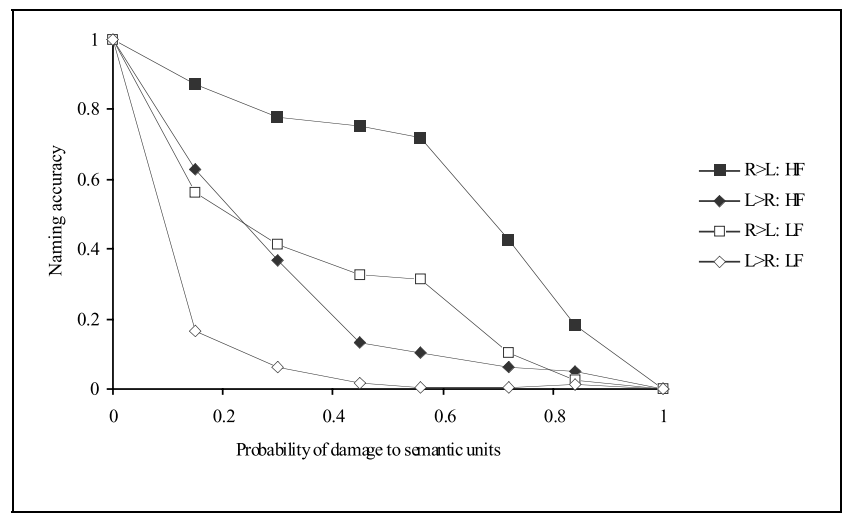

Figure 9. Effect of pattern frequency on the naming performance of model. $\mathrm{R}>\mathrm{L}-$ Simulation with more right than left semantic unit damage. $\mathrm{L}>\mathrm{R}$-Simulation with more left than right semantic unit damage. HF-Most frequent patterns. LF-Least frequent patterns.

ment (as measured by overall error rate) increases. The proportion of such errors was consistently greater for the patients with $\mathrm{R}>\mathrm{L}$ temporal atrophy than for the $\mathrm{L}>\mathrm{R}$ cases. This difference was matched qualitatively by the simulated data, though the rate for the simulation with more right than left semantic unit damage was somewhat lower than the corresponding patient data.

Irrespective of the laterality of atrophy or severity of disease, all patients with semantic dementia exhibit a strong effect of frequency/familiarity on naming performance (Lambon Ralph, Graham, Ellis, \& Hodges, 1998; Lambon Ralph, Graham, Patterson, \& Hodges, 2000; Funnell, 1995). This effect was also replicated by these simulations, where the frequency of each training pattern had a strong influence on the model's naming performance at all levels and for both distributions of damage (see Figure 9).

\section{DISCUSSION}

There is no doubt that the ability to produce the names of objects/concepts, both in connected speech and as single words, requires both intact semantic and phonological representations of the concepts and their names. One contentious issue is whether models of name production need to include an additional aspect or level of processing that intervenes between semantic and phonological levels, and that can be disrupted by brain disease or injury to produce anomia. Probably, the most typical answer to this question offered in the literature on anomia is that this additional type of deficit is required by the data. In a box-and-arrow diagram of the naming process, the deficit could presumably be assigned either to a box (the abstract word representations, or lemmas, which intervene between semantic and phonological stages in many models of speech production: Dell \& O'Seaghdha, 1992; Levelt, 1992) or to an arrow. It is important to note, from the outset, that 
this alternative hypothesis could explain the full range of behavioral data presented here by assuming that each patient had a mixture of semantic and post semantic deficits, and we acknowledge that nothing in our data rules out this interpretation. The set of neuropsychological investigations and connectionist simulations reported here suggests that it may not be necessary, however, and we shall set out below our reasons for favoring a semantic-deficit account for all of these cases. In fact, it is our working hypothesis that many other language deficits including, for example, acquired dyslexias, morphological verb impairments, and a wider range of anomic disorders, might find an account in terms of disruption to semantic and/or phonological representations alone (Lambon Ralph, Sage, \& Roberts, 2000; Joanisse \& Seidenberg, 1999; Patterson \& Lambon Ralph, 1999; Plaut et al., 1996).

Phonological deficits are not particularly germane to the present discussion: The majority of anomic patients (typically consequent upon cerebrovascular disease) do have phonological impairments (Gagnon, Schwartz, Martin, Dell, \& Saffran, 1997; Goodglass et al., 1997), but the fluent progressive aphasic patients whose anomia we have presented and simulated here do not-at least not by the measures typically used to assess this aspect of speech production (e.g., these patients essentially never make phonological errors in spontaneous speech or in single-word naming, repetition or reading; they can read and repeat nonsense words; they have normal auditory-verbal short-term memory as assessed by digit span; etc.). By contrast, the majority of these patients have quite obvious semantic deficits, at least once the condition has progressed beyond its earliest stages; and in some cases, the nature and degree of anomia is readily explicable with sole reference to the semantic degradation. Another subset of cases, however, suffer from anomia that is disproportionately severe relative to any measurable semantic impairment. This has led some researchers (including, previously, some of the authors of this article) to conclude that these latter cases have a disruption to processes between the semantic and phonological stages, and that their syndrome should be called progressive pure anomia, with the label semantic dementia reserved for the former subset of patients (Graham et al., 1995, 1998). The conclusion from the research presented here is that this fractionation is unwarranted, and that the anomia of both "subsets" can be explained by degraded conceptual knowledge. We shall now briefly summarize the reasons for this conclusion, and then address a few remaining issues.

The longitudinal patterns of naming and comprehension in 16 patients with progressive fluent aphasia/ semantic dementia replicated the two profiles previously described regarding the extent of disparity between naming and comprehension. More detailed analyses of these data established, however, that the difference between the two patterns was one of degree, not of distinct type. There was a continuum of cases that was bounded by patients like JL (Hodges et al., 1995), whose worsening anomia was coupled to a similar rate of declining comprehension, and FM (Graham et al., 1995), whose anomia was dramatically more profound than her measured comprehension deficit. Furthermore, all patients' naming errors consisted of a mixture of omissions, coordinate semantic errors, and functional descriptions, though the exact proportions again varied along a continuum: Omissions were more common for patients like FM and coordinate semantic errors for the JL-like cases.

Perhaps the most important advance of the current research is the demonstration that the two profiles are strongly associated with and predicted by the relative asymmetry of temporal lobe atrophy. Semantic dementia results from atrophy of the temporal lobes, initially most prominent at the pole and then spreading (with some variability across individual cases) to more posterior, medial, and/or superior regions in the temporal lobe, as well as to the ventromedial frontal area adjacent to the temporal pole (Mummery et al., 2000). Although simple inspection of MRI brain scans may suggest such strongly asymmetric (left/right) atrophy as to make it appear unilateral, recent techniques of quantification demonstrate that-apart from the rare case caught very early in the course of the disease-all patients have bilateral even if highly asymmetric temporal atrophy (Galton et al., in press; Mummery et al., 2000). What we have shown here is that the relative asymmetry of the temporal lobe atrophy provides an independent predictor for each patient's degree of coupling between anomia and comprehension impairment, with the disparity significantly greater in those patients with more left- than righttemporal lobe damage.

The computational simulations served to demonstrate how this factor might interact with the influence of strongly left-lateralized phonological representations. The architecture of the connectionist model was based on the twin assumptions of a bilaterally distributed network of semantic representations from which connections to phonology would be stronger for the neuroanatomically closer left-hemisphere portion of the semantic network. The potential of the right-hemisphere semantic elements to activate the phonological units directly was manipulated via the learning rate on these connections. As the relative learning rate was reduced, accurate naming performance became increasingly reliant on the integrity of the left-hemisphere semantic units and less dependent on the right (see Figure 5A). This occurred even though the two sides of the network were equivalent in their roles in supporting comprehension (see Figure 5B).

The second series of simulations compared the performance of the network and patients directly. There was a close match between the spectrum of longitudinal 
profiles observed in the patient data and the performance of the network when the functional connectivity between the right semantic and phonology units was severely restricted (to 0.01 of the general learning rate). Overall naming accuracy was highly sensitive to even small amounts of damage to the semantic units. For example, $15 \%$ damage produced a minimal drop in comprehension ( $97 \%$ correct) but more substantial and asymmetric effects on naming accuracy: $82 \%$ correct for primarily right-sided damage and 53\% for a comparable mainly left lesion. When the damage was extensive enough to result in a measurable semantic impairment (88\% correct with 30\% of the semantic units lesioned), naming was severely affected: 68\% correct for a predominantly right lesion, 33\% for left. As is evident from Figure 5A, enforced left-sided laterality of the phonological units magnified the effect of left-sided semantic damage upon naming performance; thus, when naming and comprehension were compared directly (see Figures 6 and 7), both the semantically coupled and progressive anomic patterns were reproduced as boundaries of a continuum of possible longitudinal profiles.

Although this simulation was not designed to produce all types of naming errors made by the patients (e.g., semantic superordinates such as zebra $\rightarrow$ "animal"), the network successfully captured qualitatively the different rate of semantic coordinate errors associated with the two patient subsets. The consistently greater likelihood of coordinate errors by patients with $\mathrm{R}>\mathrm{L}$ temporal lobe atrophy arises in the simulation from the division of labor between right and left semantic units. The major source of activation for the phonological layer came from the left-sided semantic elements; when these were partially disabled, not only was naming accuracy seriously compromised, but the remaining activation also was often insufficient to produce the output pattern corresponding to a semantically related exemplar. In contrast, the main consequence of lesioning the right semantic units (leaving aside the small, direct activation of phonology) was to limit the network's ability to distinguish "within-category" exemplars from one another. The resultant semantic confusion affected not only comprehension but also production due to the full interconnectivity within the semantic layer. Because many of the left semantic units remained functional (at least in the early stages of progressive damage), the network was still able to produce activation at the phonological layer; lacking sufficient differentiating information within the full semantic representation, however, the relatively efficient left semantic-to-phonology connections tended to produce semantic errors. With increasing levels of semantic unit damage, greater proportions of the left semantic units were inevitably affected and, thus, the rate of semantic errors fell, mirroring a similar decline in the patient data. The final analyses of the network's naming performance demonstrated a clear influence of pattern frequency irrespective of the distribution or severity of damage to the semantic units, reproducing the effects of frequency and familiarity on naming noted in a number of studies of semantic dementia (Lambon Ralph et al., 1998; Hirsh \& Funnell, 1995; Parkin, 1993; Patterson \& Hodges, 1992).

The two assumptions underlying the architecture of the network are worthy of brief further consideration. The first was that conceptual knowledge and, in particular, the information, which differentiates between semantically similar exemplars, is distributed across the temporal lobes bilaterally. This assumption not only squares with the semantic dementia data presented here, but also with data and hypotheses regarding other patient groups which suggest at least a degree of semantic representation in the right hemisphere, especially for concrete concepts (Saffran \& Coslett, 1998; Tranel, Damasio \& Damasio, 1997; Patterson, Vargha-Khadem, \& Polkey, 1989; Coltheart, 1981). This view does not discount the possibility that the types of knowledge supported by left- or righttemporal structures may differ, but rather assumes that differentiating information of some form is represented on both sides, and that all information summates across the bilateral neural structures resulting in one functional semantic system (Patterson \& Hodges, 2000). The central basis of the network's behavior was the additional assumption that differential connectivity from left versus right portions of the semantic network to output phonology would lead to differential involvement of left versus right semantics in naming and, thus, to the different patterns of longitudinal naming data associated with $\mathrm{R}>\mathrm{L}$ and $\mathrm{L}>\mathrm{R}$ atrophy. Although the left-right connectivity assumption adopted here is highly plausible, it is worth noting that this may not be the entire story, because the same principle could apply within the left hemisphere. That is, certain subregions within a left-sided semantic network (Vandenberghe, Price, Wise, Josephs, \& Frackowiak, 1996) may have greater anatomical proximity to, hence, stronger connections to, phonological representations.

By way of conclusion, we note that results collected from an extensive study of patients with damage to selective brain regions (Tranel et al, 1997; Damasio, Grabowski, Tranel, Hichwa, \& Damasio, 1996) have also implicated different left-right lesion distributions associated with naming and the retrieval of conceptual knowledge. Damasio et al. (1996) identified patients with deficits specific to naming persons, animals or tools and found maximal lesion overlap almost exclusively in the temporal pole, inferotemporal region and the occipital-temporal-parietal junction on the left, plus regional cerebral blood flow increases in these same areas when normal intact subjects were asked to name pictures of concrete entities. Tranel et al. (1997) 
used a recognition paradigm in which patients were required to demonstrate knowledge for the same items by providing either the correct name or defining information. Failure on this principally semantic task was associated with lesions in the right-temporal pole, rightmesial occipital-ventral temporal areas, left-mesial occipital and left-lateral occipital-temporal-parietal regions. These results would seem to fit neatly with the account given here: Impaired comprehension of concrete concepts is associated with bilateral, predominantly temporal lesions while naming deficits arise from left-sided temporal damage.

\section{METHODS}

\section{Patients}

\section{Behavioral Assessment}

Data were collected from 16 patients all of whom fulfilled the criteria for semantic dementia: anomia and impaired comprehension with relative preservation of phonology, syntax, visuospatial abilities, and day-to-day (episodic) memory. Structural brain imaging by MRI revealed focal atrophy including the polar and inferolateral aspects of the temporal lobe. Relative asymmetry in the temporal lobe atrophy was determined by a senior neurologist (JRH); in 11/16 cases, the atrophy was more pronounced in the left- than the right-temporal lobe (see below for more details). Patients were assessed longitudinally by repeated administrations of a comprehensive neuropsychological battery. The two tests that are the central focus of this article utilized the same set of 48 concrete concepts drawn from the Snodgrass and Vanderwart (1980) corpus comprising eight exemplars from each of six categories (land animals, birds, water creatures, household objects, vehicles, and musical instruments). On one occasion, the patient was presented with a line drawing of each concept and asked to provide the name. On another occasion (but close in time to the naming test), comprehension for this set was assessed using a wordpicture matching paradigm. On each trial, the patient was presented with a picture array of eight withincategory exemplars and asked to point to the picture that matched the spoken name of the target item.

\section{Assessment of Temporal Lobe Atrophy}

The degree and asymmetry of the temporal lobe atrophy was measured in more detail for 14 patients for whom suitable coronal MRI images were available, typically from a time at or shortly after initial assessment. In each patient, volumes for four regions within each temporal lobe were rated visually by a neurologist and a neuroradiologist who were blind to the patients' identity (see Galton et al., submitted). Hippocampal volume was rated using the five-point scale described in detail by
Scheltens et al. (1992). The degree of hippocampal atrophy $(0=$ normal to $4=$ severe atrophy $)$ was estimated by visual inspection of the width of the choroidal fissure, width of the temporal horn and the height of the hippocampal formation using the best slice that depicted both hippocampal formations (usually at the anterior pons). This method has shown good interrater and intrarater reliability on a dichotomized scale and has been validated against both linear and volumetric measures using different MRI sequences (Vermersch, Scheltens, Leys, \& Barkhof, 1994; Scheltens et al., 1992; Scheltens, Launer, Barkhof, Weinstein, \& van Gool, 1995). A similar four-point scale ( $0=$ normal to 3 = severe atrophy, see Galton et al., in press) was used to rate three other temporal lobe structures: (1) anterior temporal-the cerebrospinal fluid space between the sphenoid wing and anterior temporal lobe (on a representative slice before the closure of the lateral fissure); (2) medial temporal (parahippocampal gyrus) - rating the depth of the collateral sulcus on the same slice on which the hippocampus is assessed; and (3) lateral temporal-the depth of the lateral sulci on the same slice. For the present study, two values were computed from these ratings: (1) total atrophy = the average of all eight values; and (2) asymmetry = the difference between the average values for the left- and right-temporal lobes (positive values corresponded to greater left- than right-temporal lobe atrophy). There was $100 \%$ agreement between the laterality values calculated by this method and the relative asymmetry established previously by JRH.

\section{Data Analysis}

The behavioral data of the semantic dementia patients were split into two groups according to the relative asymmetry of the temporal lobe atrophy. The two sets of longitudinal word-picture matching and naming data were analyzed using two different techniques. The first grouped the data across five ranges of word-picture matching performance (less than 40\% correct; 40-70\%; 70-90\%, 90-95\%, and 95-100\%), thus, weighting the resultant average profiles in favor of those patients with the most observations. The second technique assumed that each patient's data lay on a curve that we call the NMC curve. The curve assumes an underlying variable $t$, which might be thought of as the degree of semantic integrity. The value of this variable progressively declines from a large positive to a large negative value. Naming performance relates to $t$ according to the function $\left(1+\mathrm{e}^{-t}\right)^{-1}$ and word-picture matching relates to $t$ according to the function $\left(1+\mathrm{e}^{A-t}\right)^{-1}$. When graphed against each other, these two equations produce a function for which the degree of curvature is governed by the parameter $A$. If $A=0$, then there is an equivalence between naming and comprehension (i.e., the diagonal line shown in Figures 1, 2, and 3). As 
parameter $A$ is increased the curvature becomes more extreme (see Figures 2 and 3). A value of $A$ for each patient was estimated using an iterative procedure that minimized the sum squared differences between observed and expected data. As naming was at floor for the single data point of patient PP, a value of 0.5 items was used in order to fit a NMC curve. With this second method, a tight coupling between naming and comprehension would lead to a low value of $A$, while the "progressive anomic" profile would produce higher values.

\section{Simulation}

\section{Architecture}

The network was made up from three layers of units. The initial "picture" input layer of 120 units projected activation forward to a set of 480 "semantic" units, which sent activation on to the 120 "phonology" output units. There was full connectivity between units in the input and semantic layers and between units in the semantic and phonological layers. There was no direct connection between the input and phonological layers. In addition, the units within each layer were connected to all the other units within the same layer. No hidden units were employed in these simulations. A sigmoid activation function was used for each unit with activation ranging from -1 to +1 .

\section{Training Patterns}

The network was trained using 100 abstract patterns. For the phonology layer, 100 patterns were created by setting the target activation of each bit within each output representation randomly to +0.95 or -0.95 with equal probability. Semantic representations were generated in the following way. Ten "prototype" patterns were created by setting the value of each bit to zero with a probability of .5. For nonzero bits, the target value of +0.95 or -0.95 was assigned randomly and with equal probability. Ten closely related exemplars were generated from each of the 10 prototypes by randomly switching the polarity of a small proportion of the nonzero bits-the probability of altering the value was set to .01 and the resultant exemplar representations were checked to ensure that no two were identical. The two sets of simulations used slightly different input representations. In the first, patterns were generated in exactly the same way as the phonological patternseach bit was set randomly to +0.95 or -0.95 with equal probability. While an arbitrary relationship between semantic and phonological representations seems a correct reflection of the true state of affairs (concepts with similar meanings do not have similar sounding names, and, likewise, substantial phonological overlap between two words implies nothing in the way of shared semantic features), there is a more systematic relation- ship between the visual representation of concrete exemplars and conceptual knowledge. Aspects of the visual input can provide accurate cues as to an object's identity (Lambon Ralph, Graham, et al., 2000; Caramazza, Hillis, Rapp, \& Romani, 1990) and building such relationships into connectionist networks has a significant impact on the networks' performance under damage (Lambon Ralph \& Howard, 2000; McGuire \& Plaut, 1997). For identical patterns/degrees of damage, performance is better when there is at least a partial or quasisystematic relationship. The second set of simulations, therefore, changed the mapping between input and semantic representations from an arbitrary to a quasisystematic one. This was achieved by substituting nonzero values from the first and last 60 bits from the corresponding 480-bit semantic representations into the first and last 60 bits of the input patterns. That is to say, if a semantic bit had a zero value (which it did with a probability of .5), the initial value for the input bit was maintained, otherwise, the value of the nonzero semantic bit was used as a substitute.

\section{Training}

The network was trained to produce the correct "semantic" and "phonological" representations when each of the "picture" patterns was clamped onto the input units. During every epoch of training, each of the 100 patterns was presented in a random order, and the weights (which were initially set to zero) were updated at the end of each epoch. Error-the sum squareddifference between observed and target activationswas reduced using the delta rule (McClelland \& Rumelhart, 1985). No weight decay was applied to the network. After 450 epochs, the model had learned to produce the correct semantic and phonological patterns for all 100 representations.

\section{Learning Rate}

The learning rate parameter was used for two purposes. In order to restrict the influence of the right-hemisphere semantic units on the phonological output layer, the rate of weight change in that part of the network was scaled down from the global rate of 0.005 . The effect of different degrees of reduced learning rate is shown in the first part of the Simulations. The learning rate was also scaled to investigate the influence of differential pattern frequency on the model's performance. For each prototype, each generated "exemplar" pattern was numbered from 1 to 10 . The learning rate for each was scaled by the reciprocal of its pattern number.

\section{Testing}

The network's accuracy was established using the bestfit procedure. Activation produced by the model was 
compared with the target pattern for all 100 representations. If the closest match-the pattern giving rise to the least error-was the target then the activation was counted as correct. Errors were split into "semantic" and "other" according to whether the best match was another pattern derived from the same or different "prototype." This process was completed twice; once for the semantic layer-deriving a measure for "comprehension" in the model, and once for the phonological layer-giving a measure for "naming." In order to compare comprehension performance in the model and the patients, we adopted a within-prototype best match procedure for assessment of accuracy at the semantic layer. The output for each representation was compared only against the 10 exemplars derived from the target pattern's prototype. This provides a closer analogy to the word-picture matching assessment used with the patients in which the subject is asked to select the target picture from an array of eight within-category exemplars. In fact, with a quasi-systematic relationship between input and semantic representations, all the comprehension errors produced by the model were classified as semantic. Consequently, in these circumstances, the two comprehension measures for the network produced identical results.

\section{Damage}

The network was damaged by clamping a proportion of the semantic units to zero. As most patients with semantic dementia have bilateral, though sometimes very asymmetric temporal lobe atrophy (see Introduction), the following protocol of simulated damage was used. There were six different levels of random damage: $15 \%, 30 \%, 45 \%, 56 \%, 72 \%$, and $84 \%$ of all the semantic units. Up to the $56 \%$ level, the damage was applied with an asymmetric distribution of $90 \%$ to the left and $10 \%$ to the right, or the reverse. For example, for $30 \%$ overall damage, the model was given two different lesions: $27 \%$ to the left and $3 \%$ to the right, or $3 \%$ to the left and $27 \%$ to the right. At the $72 \%$ and $84 \%$ levels of damage, the asymmetrical lesions were made to be as extreme as possible. For $72 \%$ overall damage, all of the units on one side were lesioned and $44 \%$ of the units on the other side were damaged, and for $84 \%$ overall damage, all of the units on one side and $68 \%$ of the units on the other side were lesioned. Each combination of left-right semantic lesions was repeated 10 times and the results averaged. The effect of pattern frequency was assessed by averaging across the 50 most or least frequent patterns.

\section{Acknowledgments}

We are indebted to the continuing participation of the patients included in this paper. The research was supported by funding from the National Intitutes of Health (USA) and the Medical Research Council (UK).

Reprint requests should be sent to: Dr. M.A. Lambon Ralph, University of Bristol, Department of Experimental Psychology, 8 Woodland Road, Bristol, BS8 1TN, UK; Tel: +44-117-9288545; E-mail: matt.lambon-ralf@bristol.ac.uk.

\section{Notes}

1. Although FM's naming performance dropped to zero for this set of 48 items, she was still able to produce some spontaneous speech, which remained fluent and prosodic although with a continuing dramatic decline in content-word vocabulary (see Bird, Lambon Ralph, Patterson, \& Hodges, submitted).

2. The reader may notice a slight "knee" in the curve relating naming and word-picture matching for the $\mathrm{R}>\mathrm{L}$ damage in the simulation, which is not present in the patient data. This reflects the particular assumptions that we made in the simulation about the distribution of damage. Up to the knee, $90 \%$ of the damage was directed to the right semantics and $10 \%$ was directed to the left, with the effect that there was only a very slow increase in left damage up to that point. After the knee, which represents the point at which all of the right units were completely destroyed, all further damage applied only to the left side of the model, thus, resulting in a rapid acceleration in the loss of naming performance. A similar policy was used in the $\mathrm{L}>\mathrm{R}$ simulations; in that case, the somewhat less obvious knee in the curve bends the other way.

\section{REFERENCES}

Bird, H., Lambon Ralph, M. A., Patterson, K., \& Hodges, J. R. (2000). The rise and fall of frequency and imageability: Noun and verb production in semantic dementia. Brain and Language, 73, 17-49.

Bub, D., Black, S., Hampson, E., \& Kertesz, A. (1988). Semantic encoding of pictures and words: Some neuropsychological observations. Cognitive Neuropsychology, 5, 27-66.

Caramazza, A., Hillis, A. E., Rapp, B. C., \& Romani, C. (1990). Multiple semantics or multiple confusions? Cognitive Neuropsychology, 7, 161-168.

Chertkow, H., Bub, D., Deaudon, C., \& Whitehead, V. (1997). On the status of object concepts in aphasia. Brain and Language, 58, 203-232.

Chiacchio, L., Grossi, D., Stanzione, M., \& Trojano, L. (1993). Slowly progressive aphasia associated with surface dyslexia. Cortex, 29, 145-152.

Coltheart, M. (1981). Right-hemisphere reading. Behavioral and Brain Sciences, 4, 67-68.

Damasio, H., Grabowski, T. J., Tranel, D., Hichwa, R. D., \& Damasio, A. R. (1996). A neural basis for lexical retrieval. Nature, 380, 499-505.

Dell, G. S., \& O'Seaghdha, P. G. (1992). Stages of lexical access in speech production. Cognition, 42, 287-314.

Funnell, E. (1995). Objects and properties: A study of the breakdown of semantic memory. Memory, 3, 497-518.

Gagnon, D. A., Schwartz, M. F., Martin, N., Dell, G. S., \& Saffran, E. M. (1997). The origins of formal paraphasias in aphasics' picture naming. Brain and Language, 59, 450-472.

Gainotti, G., Silveri, M. C., Daniele, A., \& Giustolisi, L. (1995). Neuroanatomical correlates of category-specific semantic disorders: A critical survey. Memory, 3, 247-264.

Galton, C. J., Gomez-Anson, B., Antoun, N., Patterson, K., Graves, M., Sahakian, B. P. S., \& Hodges, J. R. (in press). 
Differing patterns of temporal atrophy in Alzheimer's disease and semantic dementia: A new visual rating scale with volumetric validation. Journal of Neurology, Neurosurgery and Psychiatry.

Goodglass, H., Wingfield, A., Hyde, M. R., Berko Gleason, J., Bowles, N. L., \& Gallagher, R. E. (1997). The importance of word-initial phonology: Error patterns in prolonged naming efforts by aphasic patients. Journal of the International Neuropsychological Society, 3, 128-138.

Graham, K. S., Hodges, J. R., \& Patterson, K. E. (1994). The relationship between comprehension and oral reading in progressive fluent aphasia. Neuropsychologia, 32, 299-316.

Graham, K. S., Patterson, K., \& Hodges, J. R. (1995). Progressive pure anomia: Insufficient activation of phonology by meaning. Neurocase, 1, 25-38.

Graham, K. S., Patterson, K., \& Hodges, J. R. (1998). Semantic dementia and pure anomia: Two varieties of progressive fluent aphasia. In E. Visch-Brink, \& R. Bastiaanse (Eds.), Linguistic levels in aphasia (pp. 49-67). San Diego, CA: Singular Publishing Group.

Graham, K. S., Simons, J. S., Pratt, K., Patterson, K., \& Hodges, J. R. (2000). Insights from semantic dementia on the relationship between episodic and semantic memory. Neuropsychologia, 38, 313-324.

Graham, N. L., Patterson, K., \& Hodges, J. R. (in press). The emergence of jargon in progressive fluent dysgraphia: The widening gap between target and response. Cognitive Neuropsychology.

Hirsh, K. W., \& Funnell, E. (1995). Those old, familiar things: Age of acquisition, familiarity and lexical access in progressive aphasia. Journal of Neurolinguistics, 9, 23-32.

Hodges, J. R. (2000). Pick's disease: Its relationship to semantic dementia, progressive aphasia and frontotemporal dementia. In J. O'Brien, D. Ames, \& A. Burns (Eds.), Dementia. London: Arnold.

Hodges, J. R., Graham, N., \& Patterson, K. (1995). Charting the progression of semantic dementia: Implications for the organisation of semantic memory. Memory, 3, 463-495.

Hodges, J. R., \& Patterson, K. (1995). Is semantic memory consistently impaired early in the course of Alzheimer's disease? Neuroanatomical and diagnostic implications. Neuropsychologia, 33, 441-459.

Hodges, J. R., Patterson, K., Oxbury, S., \& Funnell, E. (1992). Semantic dementia: Progressive fluent aphasia with temporal lobe atrophy. Brain, 115, 1783-1806.

Joanisse, M., \& Seidenberg, M. S. (1999). Impairments in verb morphology after brain injury: A connectionist model. Proceedings of the National Academy of Sciences, U.S.A., 96, 7592-7597.

Kapur, N., Barker, S., Burrows, E. H., Ellison, D., Brice, J., Illis, L. S., Scholey, K., Colbourn, C., Wilson, B., \& Loates, M. (1994). Herpes-simplex encephalitis: Long-term magnetic-resonance-imaging and neuropsychological profile. Journal of Neurology, Neurosurgery and Psychiatry, 57, 1334-1342.

Knott, R., Patterson, K., \& Hodges, J. R. (1997). Lexical and semantic binding effects in short-term memory: Evidence from semantic memory. Cognitive Neuropsychology, 8, 1165-1216.

Lambon Ralph, M. A., Graham, K. S., Ellis, A. W., \& Hodges, J. R. (1998). Naming in semantic dementia-what matters? Neuropsychologia, 36, 775-784.

Lambon Ralph, M. A., Graham, K. S., Patterson, K., \& Hodges, J. R. (2000). Is a picture worth a thousand words? Evidence from concept definitions by patients with semantic dementia. Brain and Language, 70, 309-355.

Lambon Ralph, M. A., \& Howard, D. (2000). Gogi aphasia or semantic dementia? Simulating and assessing poor verbal comprehension in a case of progressive fluent aphasia. Cognitive Neuropsychology, 17, 437-466.

Lambon Ralph, M. A., Sage, K., \& Roberts, J. (2000). Classical anomia: A neuropsychological perspective on speech production. Neuropsychologia, 38, 186-202.

Levelt, W. J. M. (1992). Accessing words in speech production: Stages, processes and representations. Cognition, 42, $1-22$.

McClelland, J. L., \& Rumelhart, D. E. (1985). Distributed memory and the representation of general and specific information. Journal of Experimental Psychology: General, 114, 159-188.

McGuire, S., \& Plaut, D. C. (1997). Systematicity and specialization in semantics: A computational account of optic aphasia. Proceedings of the 19th annual conference of the Cognitive Science Society. Hilldale, NJ: Erlbaum.

Morton, J. (1985). Naming. In S. Newman, \& R. Epstein (Eds.), Current perspectives in dysphasia. Edinburgh: Churchill Livingstone.

Mummery, C. J., Patterson, K., Price, C. J., Ashburner, J., Frackowiak, R. S. J., \& Hodges, J. R. (2000). A voxel based morphometry study of semantic dementia: The relation of temporal lobe atrophy to cognitive deficit. Annals of $\mathrm{Neu}$ rology, 47, 36-45.

Mummery, C. J., Patterson, K., Wise, R. J. S., Price, C. J., \& Hodges, J. R. (1999). Disrupted temporal lobe connections in semantic dementia. Brain, 122, 61-73.

Parkin, A. J. (1993). Progressive aphasia without dementia: A clinical and cognitive neuropsychological analysis. Brain and Language, 44, 201-220.

Patterson, K., \& Hodges, J. R. (2000). Semantic dementia: One window on the structure and organisation of semantic memory. In L. Cermak (Ed.), Revised handbook of neuropsychology: Memory disorders. Amsterdam: Elsevier.

Patterson, K., \& Lambon Ralph, M. A. (1999). Selective disorders of reading? Current Opinion in Neurobiology, 9, 235-239.

Patterson, K., McClelland, J. L., Lambon Ralph, M. A., \& Hodges, J. R. (in press). Deficits in irregular past-tense verb morphology associated with degraded semantic knowledge. Neuropsychologia.

Patterson, K., Vargha-Khadem, F., \& Polkey, C. E. (1989). Reading with one hemisphere. Brain, 112, 39-63.

Plaut, D. C. (1999). Systematicity and specialization in semantics. In D. Heinke, G. W. Humphreys, \& A. Olson (Eds.), Connectionist models in cognitive neuroscience: Proceedings of the fifth annual neural computation and psychology workshop. New York: Springer-Verlag.

Plaut, D. C., McClelland, J. L., Seidenberg, M. S., \& Patterson, K. (1996). Understanding normal and impaired word reading: Computational principles in quasi-regular domains. Psychological Review, 103, 56-115.

Saffran, E. M., \& Coslett, H. B. (1998). Implicit vs. letter-byletter reading in pure alexia: A tale of two systems. Cognitive Neuropsychology, 15, 141-166.

Saffran, E. M., \& Schwartz, M. F. (1994). Of cabbages and things: Semantic memory from a neuropsychological perspective- a tutorial review. Attention and Performance, 25, 507-536.

Scheltens, P., Launer, L., Barkhof, F., Weinstein, H., \& van Gool, W. (1995). Visual assessment of medial temporal lobe atrophy on magnetic resonance imaging: Inter-observer reliability. Journal of Neurology, 242, 557-560.

Scheltens, P., Leys, D., Barkhof, F., Huglo, D., Einstein, H., Vermersch, P., Kuiper, M., Steinling, M., Wolters, E., \& Valk, J. (1992). Atrophy of medial temporal lobe structures on MRI in "probable" Alzheimer's disease and normal aging: Diagnostic value and neuropsychological correlates. 
Journal of Neurology, Neurosurgery and Psychiatry, 55, 967-972.

Snodgrass, J. G., \& Vanderwart, M. (1980). A standardised set of 260 pictures: Norms for name agreement, image agreement, familiarity, and visual complexity. Journal of Ex-

perimental Psychology: Human Learning and Memory, 6, 174-215.

Snowden, J. S., Goulding, P. J., \& Neary, D. (1989). Semantic dementia: A form of circumscribed cerebral atrophy. Behavioural Neurology, 2, 167-182.

Snowden, J. S., Neary, D., \& Mann, D. M. A. (1996). Frontotemporal lobar degeneration: Fronto-temporal dementia, progressive aphasia, semantic dementia. New York: Churchill Livingstone.

Tranel, D., Damasio, H., \& Damasio, A. R. (1997). A neural basis for the retrieval of conceptual knowledge. Neuropsychologia, 35, 1319-1327.

Tyler, L. K., Moss, H. E., Patterson, K., \& Hodges, J. (1997). The gradual deterioration of syntax and semantics in a patient with progressive aphasia. Brain and Language, 56, 426-476.

Vandenberghe, R., Price, C., Wise, R., Josephs, O., \& Frackowiak, R. S. J. (1996). Functional-anatomy of a common semantic system for words and pictures. Nature, 383, 254-256.

Vermersch, P., Scheltens, P., Leys, D., \& Barkhof, F. (1994). Visual rating of the hippocampal atrophy: Correlation with volumetry. Journal of Neurology, Neurosurgery and Psychiatry, 57, 1015.

Warrington, E. K. (1975). The selective impairment of semantic memory. Quarterly Journal of Experimental Psychology, 27, 635-657. 